\title{
Aggregate download throughput for TCP-controlled long file transfers in a WLAN with multiple STA-AP association rates
}

\author{
Pradeepa BK and Joy Kuri \\ Centre for Electronics Design and Technology, \\ Indian Institute of Science, Bangalore. India. \\ bpradeep@cedt.iisc.ernet.in, kuri@cedt.iisc.ernet.in
}

\begin{abstract}
We consider several WLAN stations associated at rates $r_{1}, r_{2}, \ldots, r_{k}$ with an Access Point. Each station (STA) is downloading a long file from a local server, located on the LAN to which the Access Point (AP) is attached, using TCP. We assume that a TCP ACK will be produced after the reception of $d$ packets at an STA. We model these simultaneous TCP-controlled transfers using a semi-Markov process. Our analytical approach leads to a procedure to compute aggregate download, as well as per-STA throughputs, numerically, and the results match simulations very well.
\end{abstract}

Keywords: WLAN, Association, Access Points, Infrastructure Mode.

\section{Introduction}

IEEE 802.11a/b/g/n based Wireless Local Area Networks (LANs) in "infrastructure mode" are very common in many places. In this paper, we are concerned with an analytical model for evaluating the performance of TCP-controlled downloads in a WLAN. "TCP" is the Transmission Control Protocol, which is regarded as the workhorse of the Internet; numerous applications, including web browsing, file transfer, and secure e-commerce, rely on TCP as the transport protocol. The system we consider is shown in Figure 2. A detailed analysis of the aggregate throughput of TCP-controlled file downloads for a "single rate" Access Point (AP) is given in Kuriakose et al. [10]; in this, all STAs are assumed to be associated with the AP at the same 
rate. In practice, many data rates are possible and hence considering multiple rates is important. The aggregate download throughput is evaluated for the two rates case in Krusheel and Kuri [9]. In this paper, we consider an arbitrary but finite number $k$ of possible rates of association between stations (STA) and a single AP.

We are motivated to study an analytical model because of the improved understanding that it leads to, and the useful insights that it can provide. Closed-form expressions or numerical calculation procedures are helpful because other features and capabilities can be built upon them. One possible application, which we are studying now, is to utilize the results reported here in devising a better AP-STA association policy.

Our approach is to model the number of STAs with TCP Acknowledgements (ACKs) in their Medium Access Control (MAC) queues as an embedded discrete time Markov chain (DTMC), embedded at the instants of successful transmission events. We consider a successful transmission from the AP as a reward. This leads to viewing the aggregate TCP throughput in the framework of Renewal Reward theory given in Kumar [11].

Almost the entire existing literature considers a single rate of association only. This is rather limiting, because in practice, it is extremely likely that a WLAN will have STAs associated at a number of rates allowed by the technology (for example, one of 6 Mbps, 12 Mbps, 18 Mbps, 24 Mbps, 30 Mbps, $36 \mathrm{Mbps}, 48 \mathrm{Mbps}$ or $54 \mathrm{Mbps}$ in 802.11g, and one of $1 \mathrm{Mbps}, 2$ Mbps, 5.5 Mbps or $11 \mathrm{Mbps}$ in 802.11b). A first step towards considering multiple association rates was taken in Krusheel and Kuri [9], but there, only 2 possible association rates were considered. In this paper, any number of association rates is allowed. Because of this, our model is applicable to any variant of WLAN technology, for example: $802.11 \mathrm{a} / \mathrm{b} / \mathrm{g} / \mathrm{n}$.

The contributions of this paper are as follows. We present a model for analyzing the performance of TCP-controlled file transfers with $0<k<\infty$ rates of association. This generalizes earlier work. Secondly, our model incorporates TCP-specific aspects like "delayed ACKs;" this is a technique to reduce the frequency of TCP ACK generation by a TCP receiver. In most implementations, a TCP receiver generates a TCP ACK for every $2^{\text {nd }}$ TCP packet received; our model is general, and considers that one TCP ACK is generated for every $d$ TCP packets. Our analytical results are in excellent agreement with simulations, with the discrepancy being less than $1 \%$ in all cases.

The paper is organized as follows: In Section 2, related works are dis- 
cussed. In Section 3, we state the assumptions in first part and then present our analysis. In Section 4, we present performance evaluation results. In Section 5 we discuss the results. Finally, the paper is concluded in Section 6 .

\section{Related Work}

The literature on throughput modelling in a WLAN can be classified into several groups depending on the approach.

In the first group, all WLAN entities (STAs and the AP) are assumed to be saturated, i.e., each entity is backlogged permanently. Bianchi [2], Kumar et al. [12] and Cali et al. [8] consider this saturated traffic model. However, our interest is in modelling aggregate TCP throughput, and the saturated traffic model does not capture the situation well.

To see why unsaturated traffic makes a difference, we consider Figure 1 . The left part shows a saturated traffic scenario, where all WLAN entities have packets to transmit; therefore, $(N+1)$ entities contend for the channel. The right part shows the situation with TCP in the picture. Essentially, for many TCP connections, the entire window of packets sits at the AP, leaving the corresponding STAs with nothing to send. This means that the number of contending WLAN entities is much smaller as mentioned in Kuriakose et al. [10]. This indicates why approaches relying on a model with saturated nodes are inadequate.

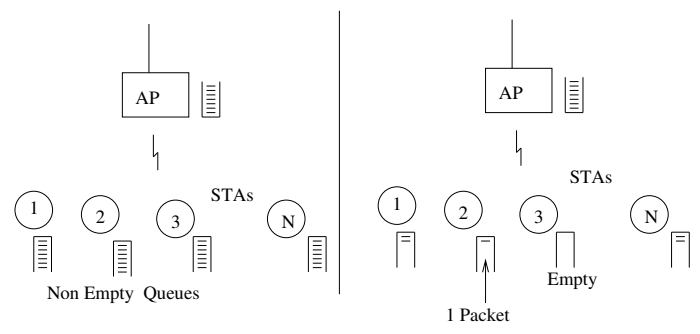

Figure 1: Two scenarios are shown: the one on the left with saturated traffic and the one on the right with unsaturated traffic. The queues of the AP and STAs are shown next to them. In the saturated traffic scenario, both the AP and the STAs have packets in their queues all the time. In the unsaturated traffic scenario which we are addressing, the AP has packets to transmit all the time but the MAC queues at STAs are empty most of the time.

The second group considers TCP traffic. Kuriakose et al. [10] propose a model for TCP-controlled file downloads in a single rate WLAN; i.e., one in 
which all STAs are associated with a single AP at the same rate. Bruno et al. [3], 44 [5], 6], [7], and Vendictis et al. [16] generalize this and analyze TCP-controlled file uploads as well as downloads; however, it is assumed again that all STAs are associated at the same rate. Similarly, Yu et al. [18] provide an analysis for a given number of STAs and a maximum TCP receive window size by using the well-known $p$-persistent model proposed in Cali et al. 8]. As noted above, these papers analyze TCP-controlled file transfers (in some cases UDP traffic is allowed as well) but limit themselves to a single rate of association.

In the third category, Bharadwaj et al. [1] consider finite AP buffers, in contrast to the previous two, where AP buffers were assumed to be infinite; however, the single rate assumption is retained.

The three groups mentioned above focus on long file transfers, where the TCP sender is assumed to have a file that is infinite in size. Miorandi et al. [13. model a different situation motivated by web browsing over a WLAN. A queuing model is proposed to compute the mean session delay for short-lived TCP flows. The impact of maximum TCP congestion window size on this delay is studied as well.

Even though a fair amount of work modelling TCP-controlled transfers has been done, we are unaware of any work that allows multiple AP-STA association rates. Clearly, this is the situation observed most often in practice, where the distance between the AP and a STA governs the rate of association. In this paper, we consider an arbitrary (but finite) number of rates of association between STAs and the AP; to the best of our knowledge, this is the first paper to consider this general model.

\section{System Model}

\subsection{Assumptions}

We consider $M$ stations associated with an AP as shown in Figure 2 , All the nodes contend for the channel using the DCF mechanism as given in IEEE $802.11 \mathrm{a} / \mathrm{b} / \mathrm{g} / \mathrm{n}$. The stations are associated with the AP at $k$ different physical rates $\left(m_{1}\right.$ STAs at rate $r_{1}, m_{2}$ STAs at rate $r_{2}, \ldots m_{k}$ STAs at rate $r_{k}$ ). We assume that there are no link errors. This is not merely a simplifying assumption; the "auto rate fallback" mechanism, implemented

widely in STAs and APs, is intended to ensure that we have an error-free but lower rate channel rather than a higher rate but error-prone channel. Thus, 
our assumption of no link errors is consistent with the usual mode of WLAN operation.

Packets in the medium are lost only due to collisions. Each station has a single TCP connection to download long files from the server and all TCP connections have equal window sizes 1 . The AP transmits TCP packets for the stations and the stations return TCP-ACK packets. Further, we assume that the AP uses the RTS-CTS mechanism while sending packets to stations and stations use basic access to send ACK packets (RTS: Request to Send, CTS: Clear to Send; these are control packets that reserve the wireless medium for the subsequent long data packet). In IEEE 802.11 WLANs, the RTS Threshold parameter determines whether the RTS-CTS exchange will precede a packet transmission. In most operational WLANs, the RTS Threshold is set such that TCP data packets are larger than the RTS Threshold, and hence sent after RTS-CTS exchange, while TCP ACK packets are smaller than the RTS Threshold, and hence sent without RTS-CTS exchange (the latter is referred to as "basic access").

Upon reception of $d$ data packets, a STA generates an ACK packet and it is enqueued at the MAC layer for transmission. We assume that all nodes have sufficiently large buffers, so that packets are not lost due to buffer overflow. Also, TCP timeouts do not occur. TCP start-up transients are ignored by considering all connections to be in Congestion Avoidance. For long file transfers (which we are considering in this paper), this is a reasonable assumption because the initial start-up phase of a TCP connection lasts for a time that is completely negligible compared to the connection lifetime; the TCP connection moves quickly to the Congestion Avoidance phase and remains there. The value of RTT is very small, since files are downloaded from a server located on the LAN as shown in Figure 2.

Thus, several TCP connections exist simultaneously and all STAs with TCP ACK packets, and the AP (which is full of TCP data packets for the STAs), contend for the channel. Since no preference is given to the AP, and it has to serve all STAs, the AP becomes a bottleneck, and it is modelled as being backlogged permanently. The aggregate throughput of the AP is shared equally among all $M$ stations.

\footnotetext{
${ }^{1}$ This can be generalized, as in Pradeepa and Kuri [15].
} 


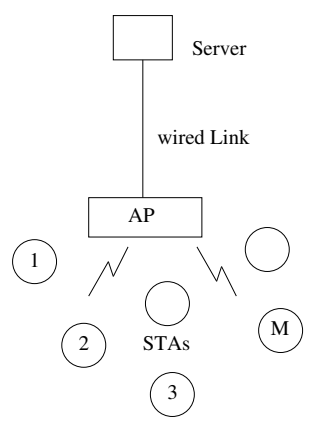

Figure 2: STAs downloading files from a server through an AP.

\subsection{Analysis}

Let $m_{i}$ be the number of stations associated with the AP at the physical transmission rate $r_{i}$, where $i \in\{1,2, \ldots k\}$ with $r_{1}>r_{2}>\ldots r_{k}$. Given that the AP wins the channel, the conditional probability that it sends a TCP data packet to a station at rate $r_{i}$ is $p_{i}$. We assume that $m_{i}$ is large. Our results will show that $m_{i} \geq 3$ or $4,1 \leq i \leq k$ (with $k \geq 4$ ) suffices for the analysis to be applicable.

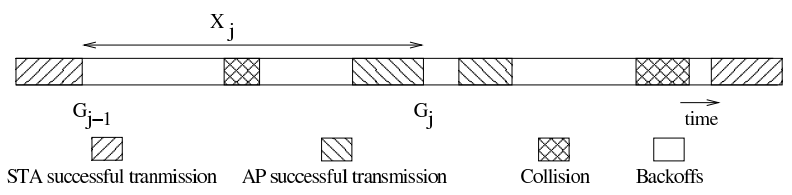

Figure 3: A possible sample path of events in WLAN shows the backoffs and the channel activity.

Figure 3 shows a possible sample path of the events on the WLAN channel. The random epochs $G_{j}$ indicate the end of the $j^{\text {th }}$ successful transmission from either the AP or one of the stations. We observe that most STAs have empty MAC queues, because, in order for many STAs to have TCP-ACK packets, the AP must have had a long run of successes - and this is unlikely because no special preference is given to the AP. So when the AP succeeds in transmitting, the packet is likely to be for a STA with an empty MAC queue.

At epoch $G_{j}$, let $S_{i, j}$ be the number of stations at rate $r_{i}$, ready with an ACK. Let $\sum_{i=1}^{k} S_{i, j}=N_{j}$ be the number of nonempty STAs. If there are $N$ nonempty STAs and a nonempty AP, each nonempty WLAN entity attempts to transmit with probability $\beta_{(N+1)}$ as in Kumar et al. [12], where 
$\beta_{N+1}$ is the attempt probability with $(N+1)$ saturated entities. It can be seen that $\left(S_{1, j}, S_{2, j}, \ldots, S_{k, j}\right)$ evolves as a Discrete Time Markov Chain (DTMC) over the epochs $G_{j}$. This allows us to consider $\left(\left(S_{1, j}, S_{2, j}, \ldots, S_{k, j}\right), G_{j}\right)$ as a Markov Renewal Sequence, and $\left(S_{1}(t), S_{2}(t), \ldots, S_{k}(t)\right)$ as a semi-Markov process.

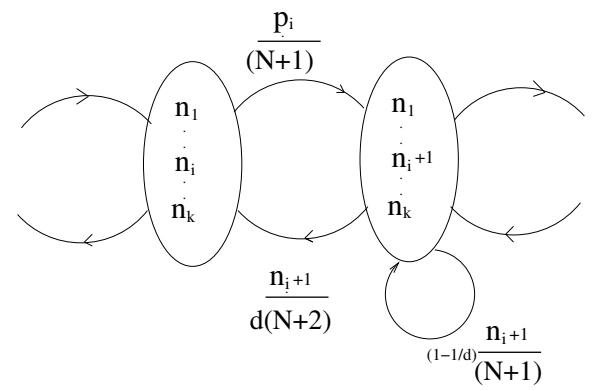

Figure 4: Embedded Markov chain formed by the AP and $n_{1}+n_{2}+\ldots+n_{k}=N$ stations associated with the AP at $k$ different data rates.

We have a multidimensional DTMC which is shown in Figure 4 transition probabilities are indicated as well. A STA generates a TCP ACK after receiving $d$ TCP packets; this is incorporated in our model in the following way. When the DTMC state is $\left(n_{1}, n_{2}, \ldots,\left(n_{i}+1\right), \ldots, n_{k}\right)$, there are $\left(n_{1}+n_{2}+\ldots\left(n_{i}+1\right)+\ldots+n_{k}+1\right)=N+2$ backlogged WLAN entities (including the AP); so, the probability that a station at rate $r_{i}$ wins the channel is $\frac{n_{i}+1}{N+2}$, since each entity is equally likely to win the contention. Further, given that a STA at rate $r_{i}$ wins the channel, the conditional probability that it will generate a TCP-ACK is $\frac{1}{d}$.

By inspection, we can say that the DTMC is irreducible; further, the Detailed Balanced Equation holds for a properly chosen set of equilibrium probabilities. The Detailed Balance Equation (DBE) is

$$
\begin{gathered}
\forall n=\left(n_{1}, n_{2}, \ldots, n_{k}\right): n_{i} \geq 0,1 \leq i \leq k, \\
\pi\left(n_{1}, n_{2}, \ldots n_{i}, \ldots n_{k}\right) \frac{p_{i}}{(N+1)}=\frac{1}{d} \pi\left(n_{1}, n_{2}, \ldots\left(n_{i}+1\right), \ldots n_{k}\right) \frac{\left(n_{i}+1\right)}{(N+2)} \\
1 \leq i \leq k
\end{gathered}
$$

Here $\pi\left(n_{1}, n_{2}, \ldots n_{i}, \ldots n_{k}\right), n_{1}, n_{2}, \ldots n_{k} \in\{0,1,2, \ldots\}$ is the stationary dis- 
tribution of the DTMC. From the set of equations given in (1) and

$$
\sum_{n_{1}=0}^{\infty} \sum_{n_{2}=0}^{\infty} \ldots \sum_{n_{k}=0}^{\infty} \pi\left(n_{1}, n_{2}, \ldots n_{i}, \ldots n_{k}\right)=1
$$

the stationary distribution is

$$
\pi\left(n_{1}, n_{2}, \ldots n_{i}, \ldots n_{k}\right)=(N+1) \Pi_{i=1}^{k} \frac{d^{n_{i}}\left(p_{i}\right)^{n_{i}}}{\left(n_{i} !\right)} * \frac{1}{(2 e)}
$$

To obtain the throughput, we use Markov regenerative analysis, culminating in the Renewal Reward Theorem given in Wolff [17], Kumar [11]. For a given state $\left(n_{1}, \ldots n_{k}\right)$, successive entries into state $\left(n_{1}, \ldots, n_{k}\right)$ form renewal epochs. To obtain the mean time between successive entries (the mean renewal cycle lengths), we obtain the mean sojourn time in the state $\left(n_{1}, \ldots n_{k}\right)$.

Let $X$ be the sojourn time in a state $\left(S_{i, j}, \ldots S_{k, j}\right)$. Conditioning on various events (idle slot, collision or successful transmission) that can happen in the next time slot, the following expression for the mean cycle length can be written down:

$$
\begin{aligned}
E_{n_{1} . . n_{k}} X & =P_{i d l e}\left(\delta+E_{n_{1} . . n_{k}} X\right)+\Sigma_{i}\left(P_{s A P}^{r_{i}} T_{s A P}^{r_{i}}\right)+\Sigma_{i}\left(P_{c}^{r_{i}}\left(T_{c}^{r_{i}}+E_{n_{1} . . n_{k}} X\right)\right) \\
& +\Sigma_{i}\left(P_{s S T A}^{r_{i}} T_{s S T A}^{r_{i}}\right)+\Sigma_{i}\left(P_{c S T A}^{r_{i}}\left(T_{c S T A}^{r_{i}}+E_{n_{1} . . n_{k}} X\right)\right)
\end{aligned}
$$

In the above expression (3), $P_{\text {idle }}$ is the probability of the slot being idle, $P_{s A P}^{r_{i}}$ is the probability that the AP wins the contention and transmits the data packet at rate $r_{i}$ and $P_{s S T A}^{r_{i}}$ is the probability that a STA associated at rate $r_{i}$ wins the channel ("s" in the suffix stands for "success"). Correspondingly, the conditional expected sojourn times in state $\left(n_{1} . . n_{k}\right)$, given the events are, respectively, $\left(\delta+E_{n_{1} . . n_{k}} X\right), T_{s A P}^{r_{i}}$ and $T_{s S T A}^{r_{i}}$. Detailed expressions for these quantities are provided in the Appendix.

The $3^{\text {rd }}$ and $5^{\text {th }}$ terms on the right side of $(3)$ correspond to collision events. The third term in (3) arises when the AP transmits a TCP data packet to a station at rate $r_{i}$ and some other stations are involved in a collision; in other words, the third term captures the situations in which the AP is involved in a collision. The fifth term in (3) captures collision events in which the AP is not involved; we have a STA transmitting a TCP ACK packet to the AP at rate $r_{i}$ and one or more other STAs transmitting 
simultaneously. The various probabilities have been obtained by using the attempt probability $\beta_{N+1}$, when there are $(N+1)$ contending nodes. From Equation (3) we have $E_{n_{1} . . n_{k}} X=$

$$
\frac{P_{i d l e}+\sum P_{s A P}^{r_{i}} T_{s A P}^{r_{i}}+\sum P_{c}^{r_{i}} T_{c}^{r_{i}}+\sum P_{s S T A}^{r_{i}} T_{s S T A}^{r_{i}}+\sum P_{c S T A}^{r_{i}} T_{c S T A}^{r_{i}}}{1-P_{i d l e}-\sum P_{c}^{r_{i}}-\sum P_{c S T A}^{r_{i}}}
$$

We are interested in finding the long run time average of successful transmissions from the AP. We obtain this by applying the Renewal Reward Theorem of Wolf [17]. To get the mean renewal cycle length, we can use the mean sojourn time given in Equation (4) and use Theorem 5.3 in [11]. The mean reward in a cycle can be obtained as follows. A reward of 1 is earned when the AP transmits a TCP data packet successfully by winning the channel. The probability of the AP winning the channel is $\frac{1}{\left(n_{1}+n_{2}+\ldots n_{k}+1\right)}$. Similarly, a reward of 0 is earned with probability $\left(1-\frac{1}{\left(n_{1}+n_{2}+\ldots n_{k}+1\right)}\right)$. Therefore, the expected reward is $\frac{1}{\left(n_{1}+n_{2}+\ldots n_{k}+1\right)}$.

Putting all this together, the aggregate TCP throughput can be calculated as 11]

$$
\Phi_{A P-T C P}=\frac{\sum_{n_{1}=0}^{\infty} \Sigma_{n_{2}=0}^{\infty} \ldots \Sigma_{n_{k}=0}^{\infty} \pi\left(n_{1} \ldots n_{k}\right) \frac{1}{n_{1}+\ldots+n_{k}+1}}{\sum_{n_{1}=0}^{\infty} \Sigma_{n_{2}=0}^{\infty} \ldots \Sigma_{n_{k}=0}^{\infty} \pi\left(n_{1} \ldots n_{k}\right) E_{n_{1}, . . n_{k}} X}
$$

\section{Evaluation}

To verify the accuracy of the model, we performed experiments using the Qualnet 4.5 network simulator [14]. We considered 802.11b physical data rates: $1 \mathrm{Mbps}, 2 \mathrm{Mbps}, 5.5 \mathrm{Mbps}$ and $11 \mathrm{Mbps}$; higher rates correspond to smaller distances between the STAs and the AP. In Table 1, results are given for a few cases of this multirate scenario. For example, the first row considers a total of 10 STAs associated with the AP, out of which 2 STAs are associated at $11 \mathrm{Mbps}$ and $2 \mathrm{Mbps}$ respectively, while 3 STAs are associated at 5.5 Mbps and $1 \mathrm{Mbps}$, respectively. The values of $p_{i}$ in Equation (2) are calculated by using the number of STAs associated with the AP. For example, in the first row in Table 1, $p_{1}$ (for $11 \mathrm{Mbps}$ ) is $\frac{2}{10}$.

In $802.11 \mathrm{~g}$, the different possible data rates are 54, 48, 36, 24, 18, 12 and $6 \mathrm{Mbits} / \mathrm{s}$. Qualnet 4.5 is configured to this mode by setting the channel frequency for the 802.11 a radio as $2.4 \mathrm{GHz}$. In Table 2, comparisons between analytical and simulation values are given. 


\begin{tabular}{|c|c|c|c|c|c|c|c|}
\hline & \multicolumn{6}{|c|}{ No. of STAs at rate (Mbps) } & \multicolumn{3}{c|}{ Aggregate Throughput (Mbps) } \\
\hline $\mathrm{M}$ & 11 & 5.5 & 2 & 1 & Analysis & Simulation & Error \% \\
\hline \multirow{2}{*}{10} & 2 & 3 & 2 & 3 & 1.0569 & 1.0492 & 0.80 \\
& 1 & 2 & 3 & 4 & 0.8397 & 0.8329 & 0.81 \\
\hline \multirow{2}{*}{12} & 2 & 2 & 4 & 4 & 0.9167 & 0.9093 & 0.81 \\
& 4 & 4 & 2 & 2 & 1.4667 & 1.4549 & 0.80 \\
\hline
\end{tabular}

Table 1: Analysis and Simulation Results [Mbps] for multirate AP in IEEE 802.11b.

\begin{tabular}{|c|c|c|c|c|c|c|c|c|c|}
\hline & \multicolumn{6}{|c|}{ No. of STAs at rate (Mbps) } & \multicolumn{3}{|c|}{ Aggregate Throughput (Mbps) } \\
\hline $\mathrm{M}$ & 54 & 48 & 36 & 24 & 18 & 6 & Analysis & Simulation & Error\% \\
\hline \multirow{6}{*}{15} & 1 & 2 & 3 & 4 & 2 & 3 & 8.14 & 8.18 & 0.49 \\
& 2 & 1 & 3 & 4 & 2 & 3 & 8.16 & 8.20 & 0.48 \\
& 3 & 2 & 1 & 4 & 2 & 3 & 8.31 & 8.32 & 0.12 \\
& 4 & 3 & 2 & 1 & 3 & 2 & 10.22 & 10.25 & 0.29 \\
& 3 & 2 & 4 & 3 & 1 & 2 & 10.38 & 10.41 & 0.29 \\
& 3 & 2 & 4 & 3 & 2 & 1 & 12.27 & 12.31 & 0.24 \\
\hline
\end{tabular}

Table 2: Throughput [Mbps] of multirate AP by analysis and simulation for IEEE $802.11 \mathrm{~g}$.

\begin{tabular}{|c|c|c|c|c|c|c|c|}
\hline & \multicolumn{6}{|c|}{ No. of STAs at rate (Mbps) } & \multicolumn{3}{c|}{ Aggregate Throughput (Mbps) } \\
\hline M & 11 & 5.5 & 2 & 1 & Analysis & Simulation & Error \% \\
\hline \multirow{2}{*}{10} & 2 & 3 & 2 & 3 & 1.1221 & 1.1131 & 0.80 \\
& 1 & 2 & 3 & 4 & 0.8889 & 0.8814 & 0.81 \\
\hline \multirow{2}{*}{12} & 2 & 2 & 4 & 4 & 0.9715 & 0.9637 & 0.81 \\
& 4 & 4 & 2 & 2 & 1.5647 & 1.5523 & 0.80 \\
\hline
\end{tabular}

Table 3: Analysis and Simulation Results [Mbps] for multirate AP in IEEE 802.11b. Delayed ACKs are implemented at the receiver with alternate segment being acknowledged $(d=2)$.

In Tables 3 , and 4 comparisons between analytical and simulation values are given for TCP with delayed ACKs. We observe that in all cases, the analytical results are in excellent agreement with simulations. 


\begin{tabular}{|c|c|c|c|c|c|c|c|c|c|}
\hline & \multicolumn{6}{|c|}{ No. of STAs with at (Mbps) } & \multicolumn{3}{|c|}{ Aggregate Throughput (Mbps) } \\
\hline M & 54 & 48 & 36 & 24 & 18 & 6 & Analysis & Simulation & Error\% \\
\hline \multirow{6}{*}{15} & 1 & 2 & 3 & 4 & 2 & 3 & 8.14 & 8.18 & 0.49 \\
& 2 & 1 & 3 & 4 & 2 & 3 & 8.16 & 8.20 & 0.48 \\
& 3 & 2 & 1 & 4 & 2 & 3 & 8.31 & 8.32 & 0.12 \\
& 4 & 3 & 2 & 1 & 3 & 2 & 10.22 & 10.25 & 0.29 \\
& 3 & 2 & 4 & 3 & 1 & 2 & 10.38 & 10.41 & 0.29 \\
& 3 & 2 & 4 & 3 & 2 & 1 & 12.27 & 12.31 & 0.24 \\
\hline
\end{tabular}

Table 4: Throughput [Mbps] of multirate AP by analysis and simulation for IEEE $802.11 \mathrm{~g}$. Delayed ACKs are implemented at the receiver with alternate segment being acknowledged $(d=2)$.

\section{Discussion}

In this work, we presented an analytical model to obtain the aggregate throughput when several TCP-controlled long file downloads are going on. Now let us consider simultaneous TCP uploads and downloads. The attempt behaviour of nodes is independent of the packet length. If we interchange downlink data packets sent by the AP with ACK packets and uplink ACK packets sent by stations with the TCP data packets, the same analysis holds good for the TCP-controlled file uploads.

Another case arises when some stations are uploading and some are downloading long files. Here also our basic Markov model for number of stations with packets to send remains the same, if all the TCP windows are equal. Even different window sizes can be taken care of by this approach. Some of these extensions have been analyzed in [15].

In our simulation and numerical evaluation, we used the $802.11 \mathrm{~b}$ and 802.11g standards. However, our mathematical expressions are independent of these standards, and hence the model can be applied to any other standard that has different number of physical data rates.

\section{Conclusion}

In this work, we have presented a simple analytical model for the aggregate throughput for TCP-controlled long file transfers in a "multirate" AP. We verified the correctness of the analytical model with the simulation results. As future work, we plan to consider short file transfers. This can 
be used to estimate the delay seen by stations. Further, association schemes can be built upon this.

\section{References}

[1] Bhardwaj, O., Sharma, G. V. V., Panda, M., and Kumar, A. (2009). "Modelling finite buffer on TCP traffic over an IEEE 802.11 infrastructure WLAN," Computer Networks an Elsevier journal, Vol.53, 2855-2869.

[2] Bianchi, G. (2000). "Performance analysis of the IEEE 802.11 distributed coordination function," IEEE JSAC, vol. 18, no. 3, 535547.

[3] Bruno, R., Conti, M., and Gregori, E. (2004). "Throughput Analysis of TCP clients in Wi-Fi Hot Spot Networks," Networking 2004, LNCS 2042, pages 626-637.

[4] Bruno, R., Conti, M., and Gregori, E. (2008). "Throughput analysis and measurements in IEEE 802.11 WLANs with TCP and UDP traffic flows," IEEE Transactions on Mobile Computing, vol.7, no.2, 171-186.

[5] Bruno, R., Conti, M., and Gregori, E. (2008). "An accurate closed-form formula for the throughput of long-lived TCP connections in IEEE 802.11 WLANs," Computer Networks, vol. 52, 199-212.

[6] Bruno, R., Conti, M., and Gregori, E. (2009). "Average value analysis of 802.11 WLANs with persistent TCP flows," IEEE Communication Letters, vol.13, no.4.

[7] Bruno, R., Conti, M., and Gregori, E. (2006). "Performance modelling and measurements of TCP transfer throughput in 802.11-based WLAN," Proceedings of the 9th ACM international symposium on Modeling analysis and simulation of wireless and mobile systems MSWiM' 06 .

[8] Cali, F., Conti, M., and Gregori, E. (2000). "Dynamic Tuning of the IEEE 802.11 protocol to achieve a Theoretical Throughput Limit," IEEE/ACM Transaction On Networking, vol. 8 no. 6 pp. 785-799.

[9] Krusheel M and J. Kuri. (2009). "Performance Analysis of TCP Uploads in WLANs with Multiple Rates," National Conference on Communications. 
[10] Kuriakose, G., Harsha, S. , Kumar, A., and Sharma, V. (2006). "Analytical models for capacity estimation of IEEE 802.11 WLANs using DCF for internet applications," Wireless Networks Springer 15 (2).

[11] Kumar, A. "Discrete Event Stochastic Processes and Queueing Theory: Lecture Notes for an Engineering Curriculum,". http://www.ece.iisc.ernet.in/ anurag/Books.htm.

[12] Kumar, A., Altman, E., Miorandi, D., and Goyal, M. (2007). "New insights from a fixed point analysis of single cell IEEE 802.11 WLANs," IEEE/ACM Transactions on Networking, Vol. 15, No 3, pp. 588-601.

[13] Miorandi, D., Kherani, A., and Altman, E. (2006). "A queueing model for HTTP traffic over IEEE 802. 11 WLANs," Computer Networks, vol. 50, no. 1, pp. 63-79.

[14] Qualnet Simulator, www.scalable-networks.com.

[15] Pradeepa, BK., and Kuri, J. , (2011). "Aggregate AP Throughputs for Long File Transfers in a WLAN controlled by Inhomogeneous TCP Connections," Proceeding of International Conference on Communication and Signal Processing, 2011. http://arxiv.org/abs/1009.0919.

[16] Vendictis, A. D., Vacirca, F., and Baiocchi, A., (2005). "Experimental Analysis of TCP and UDP Traffic Performance over Infra-structured 802.11b WLANs," Proceedings of the European Wireless.

[17] Wolff, R.W. (1989). "Stochastic Modeling and the Theory of Queues," Prentice Hall .

[18] Yu, J., Choi, S., (2007). "Modeling and analysis of TCP dynamics over IEEE 802.11 WLAN," Proceedings of the IFIP WONS,, pp. 154-161 Obergurgl, Austria. 


\section{Appendices}

Expressions for probabilities and times discussed in Section 3.2

$P_{\text {idle }}$ is the probability of the slot being idle.

$=\left(1-\beta_{N+1}\right)^{N+1}$

$P_{s A P}^{r_{i}}$ is probability that the AP wins the channel and transmits a data packet at rate $r_{i}$ (i.e., to a rate $\left.r_{i} \mathrm{STA}\right)$.

$=p_{i} \beta_{N+1}\left(1-\beta_{N+1}\right)^{N}$

$P_{c}^{r_{i}}$ is the probability of the collision event which involves the AP and STAs, with the AP transmitting to a rate $r_{i}$ STA

$=p_{i} \beta_{N+1}\left(1-\left(1-\beta_{N+1}\right)^{N}\right)$

$P_{s S T A}^{r_{i}}$ is the probability that an STA at rate $r_{i}$ wins the channel $=n_{i} \beta_{N+1}\left(1-\beta_{N+1}\right)^{N}$

$P_{C S T A}^{r_{i}}$ is the probability of a collision event involving only STAs at rate $r_{i}$ and higher. Here, the event we are interested in is as follows. One STA at rate $r_{i}$ transmits AND at least one other STA at rate $r_{i}$ or higher transmits AND no STA at rate $r_{i+1}$ or lower transmits. This probability is obtained as follows. We consider two cases: (a) 2 or more rate $r_{i}$ STAs transmit, and we do not care about whether any higher rate STA transmits or not; (b) Exactly one rate $r_{i}$ STA transmits AND at least one higher rate STA transmits. Then, the probability is

$$
\begin{aligned}
& {\left[\left(1-\left(1-\beta_{N+1}\right)^{n_{i}}-n_{i} \beta_{N+1}\left(1-\beta_{N+1}\right)^{n_{i}-1}\right)\right.} \\
& \left.+n_{i} \beta_{N+1}\left(1-\beta_{N+1}\right)^{n_{i}-1}\left(1-\left(1-\beta_{N+1}\right)^{n_{1}+n_{2}+\ldots+n_{i}-1}\right)\right] \times \\
& \left(1-\beta_{N+1}\right)^{n_{i+1}+n_{i+2}+\ldots+n_{k}+1}
\end{aligned}
$$

To verify that the sum of all the probabilities is 1 :

$$
\begin{aligned}
& P_{\text {idle }}+\sum_{i=1}^{k} P_{s A P}^{r_{i}}+\sum_{i=1}^{k} P_{c}^{r_{i}}+\sum_{i=1}^{k} P_{s S T A}^{r_{i}}+\sum_{i=1}^{k} P_{c S T A}^{r_{i}} \\
& =\left(1-\beta_{N+1}\right)^{N+1}+\beta_{N+1}\left(1-\beta_{N+1}\right)^{N}+N \beta_{N+1}\left(1-\beta_{N+1}\right)^{N} \\
& +\beta_{N+1}\left(1-\left(1-\beta_{N+1}\right)^{N}\right)+\sum_{i=1}^{k}\left(1-\beta_{N+1}\right)^{n_{i+1}+\ldots+n_{k}+1} \\
& -\sum_{i=1}^{k}\left(1-\beta_{N+1}\right)^{n_{i}+\ldots+n_{k}+1}-\sum_{i=1} k n_{i} \beta_{N+1}\left(1-\beta_{N+1}\right)^{N} \\
& =\left(1-\beta_{N+1}\right)^{N+1}+\beta_{N+1}\left(1+N\left(1-\beta_{N+1}\right)^{N}\right) \\
& +\left(1-\beta_{N+1}\right)-\left(1-\beta_{N+1}\right)^{N+1}-N \beta_{N+1}\left(1-\beta_{N+1}\right)^{N+1} \\
& =1
\end{aligned}
$$


From the above verification, it is clear that all possibilities events have been considered in Equation (3).

$T_{c}^{r_{i}} \quad$ is the collision duration when the AP and STAs at rate $r_{i}$ are involved.

$=T_{p}+T_{P H Y}+\frac{L_{M A C}+L_{I P H}+L_{T C P-A C K}}{r_{i}}+T_{E I F S}$

$T_{s A P}^{r_{i}}$ is the time taken by AP to send a packet to an STA at rate $r_{i}$

$=T_{p}+T_{P H Y}+\frac{L_{R T S}}{C_{c}}+T_{S I F S}+T_{p}+T_{P H Y}+\frac{L_{C T S}}{C_{c}}+$ $T_{S I F S}+T_{p}+T_{P H Y}+\frac{L_{M A C}+L_{I P H}+L_{T C P H}+L_{T C P}}{r_{i}}+T_{S I F S}+$ $T_{p}+T_{P H Y}+\frac{L_{A C K}}{C_{c}}+T_{D I F S}$

$T_{s S T A}^{r_{i}}$ is the time required to transmit one TCP-ACK packet from an STA at rate $r_{i}$, including overhead

$=T_{p}+T_{P H Y}+L_{M A C}+\frac{L_{I P H}+L_{T C P-A C K}}{r_{i}}+T_{S I F S}+T_{p}+$ $T_{P H Y}+\frac{L_{A C K}}{r_{i}}+T_{D I F S}$

$T_{C S T A}^{r_{i}}$ is the collision duration of STAs at rate $r_{i}$ $=T_{p}+T_{P H Y}+\frac{L_{M A C}+L_{I P H}+L_{T C P-A C K}}{r_{i}}+T_{E I F S}$

The values of $T_{p}, T_{P H Y}, L_{M A C}, L_{I P H}, L_{T C P-A C K}, T_{D I F S}, T_{S I F S}$, and $T_{E I F S}$ are standard dependent, and are mentioned in Kuriakose et al. [10], and Krusheel et al. [9]. The values of these parameters are given in Table .5. 


\begin{tabular}{|c|c|c|c|}
\hline Parameters & Symbol & $802.11 \mathrm{~b}$ & $802.11 \mathrm{~g}$ \\
\hline \hline Max PHY data rate & $r_{d}$ & $11 \mathrm{Mbps}$ & $54 \mathrm{Mbps}$ \\
\hline Control rate & $r_{c}$ & $2 \mathrm{Mbps}$ & $6 \mathrm{Mbps}$ \\
\hline PLCP preamble time & $T_{p}$ & $144 \mu s$ & \\
\hline PHY Header time & $T_{P H Y}$ & $48 \mu s$ & $20 \mu s$ \\
\hline MAC Header size & $L_{M A C}$ & 34 bytes & 34 bytes \\
\hline RTS Header size & $L_{R T S}$ & 20 bytes & 20 bytes \\
\hline CTS Header size & $L_{C T S}$ & 14 bytes & 14 bytes \\
\hline MAC ACK Header size & $L_{A C K}$ & 14 bytes & 14 bytes \\
\hline IP Header size & $L_{I P H}$ & 20 bytes & 20 bytes \\
\hline TCP Header size & $L_{T C P H}$ & 20 bytes & 20 bytes \\
\hline TCP ACK Packet size & $L_{T C P-A C K}$ & 20 bytes & 20 bytes \\
\hline TCP data payload size & $L_{T C P}$ & 1460 bytes & 1460 bytes \\
\hline System slot time & $\delta$ & $20 \mu s$ & $9 \mu s$ \\
\hline DIFS time & $T_{D I F S}$ & $50 \mu s$ & $28 \mu s$ \\
\hline SIFS time & $T_{S I F S}$ & $10 \mu s$ & $10 \mu s$ \\
\hline EIFS time & $T_{E I F S}$ & $364 \mu s$ & $364 \mu s$ \\
\hline CWmin & CWmin & 31 & 15 \\
\hline CWmax & CWmin & 1023 & 1023 \\
\hline
\end{tabular}

Table .5: Values of Parameters used in Analysis and Simulation 